\title{
Comparison of Visual, Refractive and Ocular Surface Outcomes Between Small Incision Lenticule Extraction and Laser-Assisted In Situ Keratomileusis for Myopia and Myopic Astigmatism
}

\author{
Yumi Tsz-Ying Lau - Kendrick Co Shih • Ryan Hin-Kai Tse • \\ Tommy Chung-Yan Chan · Vishal Jhanji
}

Received: June 12, 2019 / Published online: July 19, 2019

(C) The Author(s) 2019

\begin{abstract}
Laser-assisted in situ keratomileusis (LASIK) is the most commonly performed laser refractive surgical technique worldwide for the treatment of myopia and myopic astigmatism. In recent years, small incision lenticule extraction (SMILE) has emerged as a promising alternative to LASIK, requiring only a single femtosecond laser to create an intrastromal lenticule, which is then removed via a small incision. The technique obviates the need for a corneal flap. A number of published studies have compared the two techniques in terms of
\end{abstract}

Enhanced digital features To view enhanced digital features for this article go to https://doi.org/10.6084/ m9.figshare.8845133.

Y. T.-Y. Lau · K. C. Shih $(\bowtie) \cdot$ R. H.-K. Tse

Department of Ophthalmology, Li Ka Shing Faculty of Medicine, University of Hong Kong, Hong Kong SAR, China

e-mail: kcshih@hku.hk

T. C.-Y. Chan

Department of Ophthalmology, Hong Kong

Sanatorium and Hospital, Hong Kong SAR, China

T. C.-Y. Chan

Department of Ophthalmology and Visual Sciences, The Chinese University of Hong Kong, Hong Kong SAR, China

V. Jhanji

Department of Ophthalmology, University of

Pittsburgh Medical Center, Pittsburgh, USA visual, refractive and ocular surface outcomes. This review compares the clinical outcomes between LASIK and SMILE in treating myopia and myopic astigmatism based on studies published in the last 5 years. Twenty-two studies were included, all of which were observational in nature. Results suggest that the two techniques have comparable visual outcomes in terms of safety, efficacy and predictability, although recovery in visual acuity may be slower in SMILE-treated than LASIKtreated eyes. SMILE is found to result in less severe postoperative dry eye symptoms and faster recovery of corneal sensitivity than LASIK. It is important to note, however, that the SMILE technique is limited by the lack of a cyclotorsion-compensation system and option for customized treatment profile. The heterogeneity of results in this review may be attributable to the use of different LASIK platforms in different studies. Few studies compared the outcomes regarding severity of myopia. Future prospective randomized controlled trials with a larger sample size and longer follow-up period will be highly beneficial for progress in this field.

Keywords: Astigmatism; Corneal sensitivity; Dry eye; Laser refractive surgery; LASIK; Myopia; SMILE 


\section{INTRODUCTION}

Laser-assisted in situ keratomileusis (LASIK) is a popular surgical procedure for the treatment of refractive errors. It involves the creation of a corneal flap, initially through the use of a microkeratome, and subsequently through a femtosecond laser, followed by excimer laser treatment on the uncovered cornea stromal surface [1]. More than 16 million LASIK surgeries have been performed worldwide since the 1990s, and the safety, efficacy and predictability of the procedure have been firmly established in existing literature. It remains the gold standard of laser refractive surgery in terms of visual outcomes, refractive predictability and patient recovery [2]. The emergence of small incision lenticule extraction (SMILE) in the last decade has provided an alternative for patients considering cornea laser refractive surgery for myopia and myopic astigmatism. Since its introduction in 2011, SMILE has quickly gained attention as a promising approach requiring only a single laser treatment. Here, a cornea intrastromal lenticule of predetermined shape and thickness is created by the VisuMax femtosecond laser (Carl Zeiss AG, Germany) and then extracted through a small corneal incision [3]. This flapless technique is therefore theoretically less damaging to the ocular surface than LASIK and other corneal surface ablation techniques. The VisuMax platform was approved by the United States Food and Drug Administration (USFDA) on October 4, 2018, for SMILE treatment of myopia up to $-10.0 \mathrm{D}$ in power and myopic astigmatism up to $-3.00 \mathrm{D}$ in power, for a total spherical equivalent (SE) of no more than $-10.0 \mathrm{D}$. In comparison, a number of excimer lasers are approved by the USFDA for LASIK treatment of myopia up to $-8.0 \mathrm{D}$ and myopic astigmatism up to $-3.00 \mathrm{D}$ in power, for a total SE of no more than 9.0 D. The lower maximum spherical treatment in LASIK versus SMILE is because of the need for a cornea flap in the former.

Despite being theoretically less invasive, SMILE has its disadvantages when compared with LASIK. While there are LASIK platforms equipped with cyclotorsion error compensation and eye-tracking systems $[4,5]$, these mechanisms are currently absent in the VisuMax femtosecond laser platform [6]. The SMILE technique also lacks the option for a customized treatment profile. SMILE is thus theoretically inferior to LASIK in terms of refractive outcomes.

A number of studies comparing the clinical and refractive outcomes between LASIK and SMILE in different populations have been published over recent years. While some have reported the clinical superiority of SMILE, others have demonstrated that the two techniques result in similar outcomes. Based on the existing literature, this review aims to summarize the latest evidence on SMILE and LASIK outcomes from published research. We will focus on the following outcomes: visual acuity, higher-order aberrations (HOAs), corneal sensitivity and ocular surface outcomes.

\section{METHODS}

A literature search was performed on Entrez PubMed on September 26, 2018. Search keywords including "LASIK", "SMILE" and "myopia" were used. From the initial search, 84 studies were identified. The search was limited to English-language original articles on human studies that were available in full text and published within the last 5 years (with a search interval of October 2013 to September 2018). The remaining studies were further reviewed for subject relevance based on article abstracts and full texts. Criteria for relevance included, but were not limited to, patient-oriented parameters including visual outcomes, HOAs, corneal sensitivity and ocular surface disease. For example, studies comparing SMILE and LASIK techniques in ex vivo cadaveric eyes were not included due to the lack of clinical outcomes. A total of 22 studies were included in this review after curation, all of which were observational studies. This article is based on previously conducted studies and does not contain any studies with human participants or animals performed by any of the authors. 


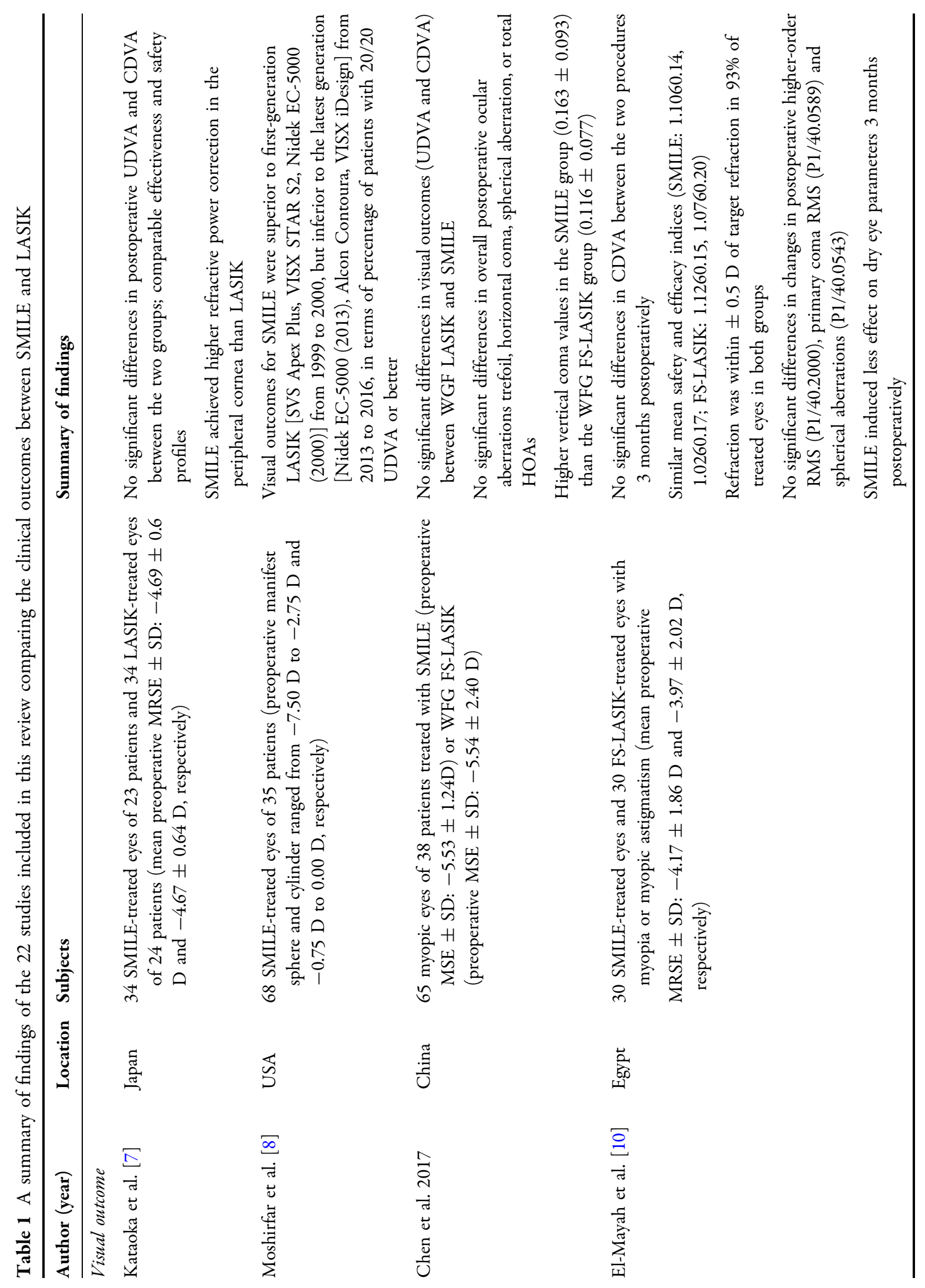




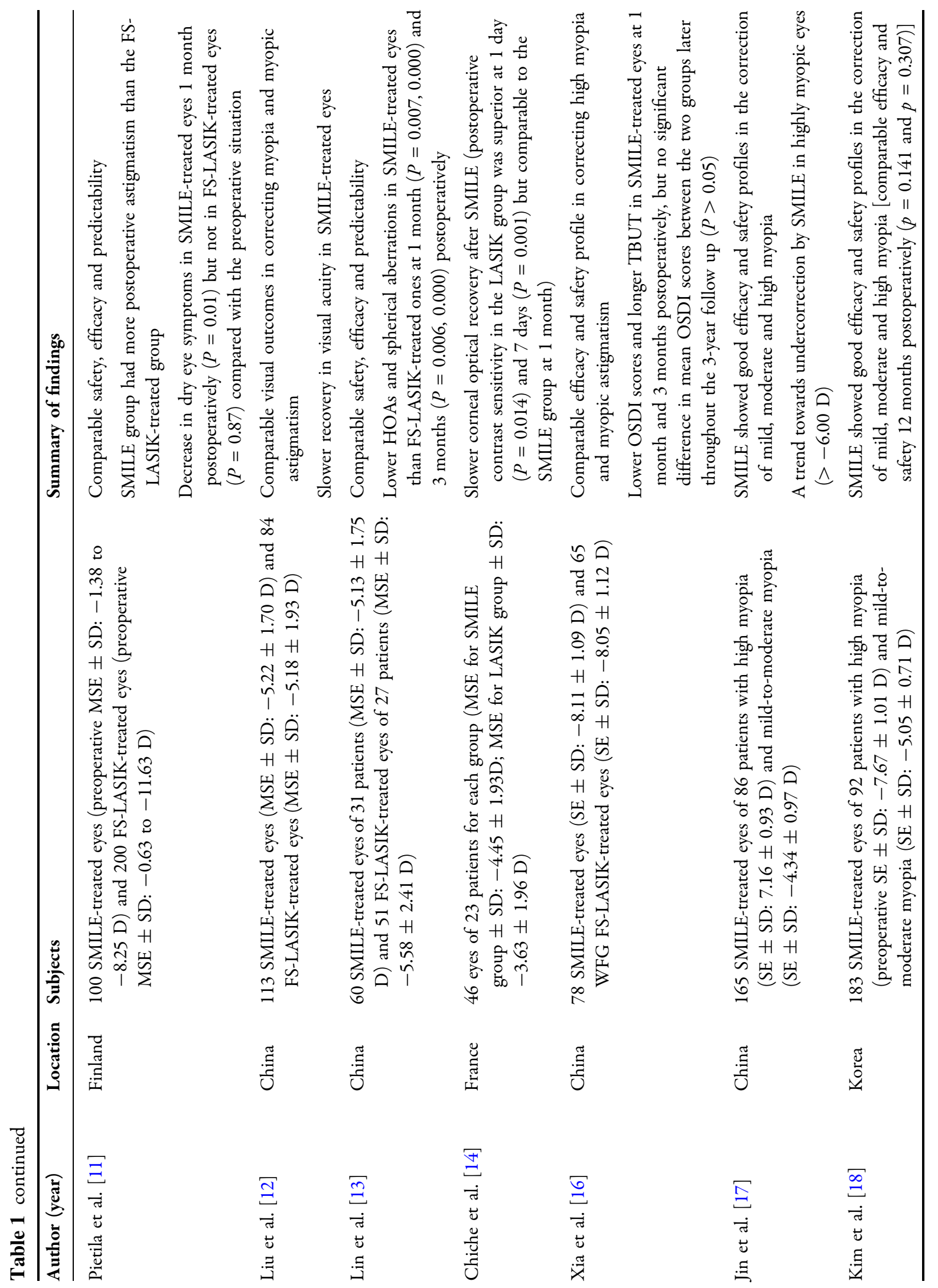




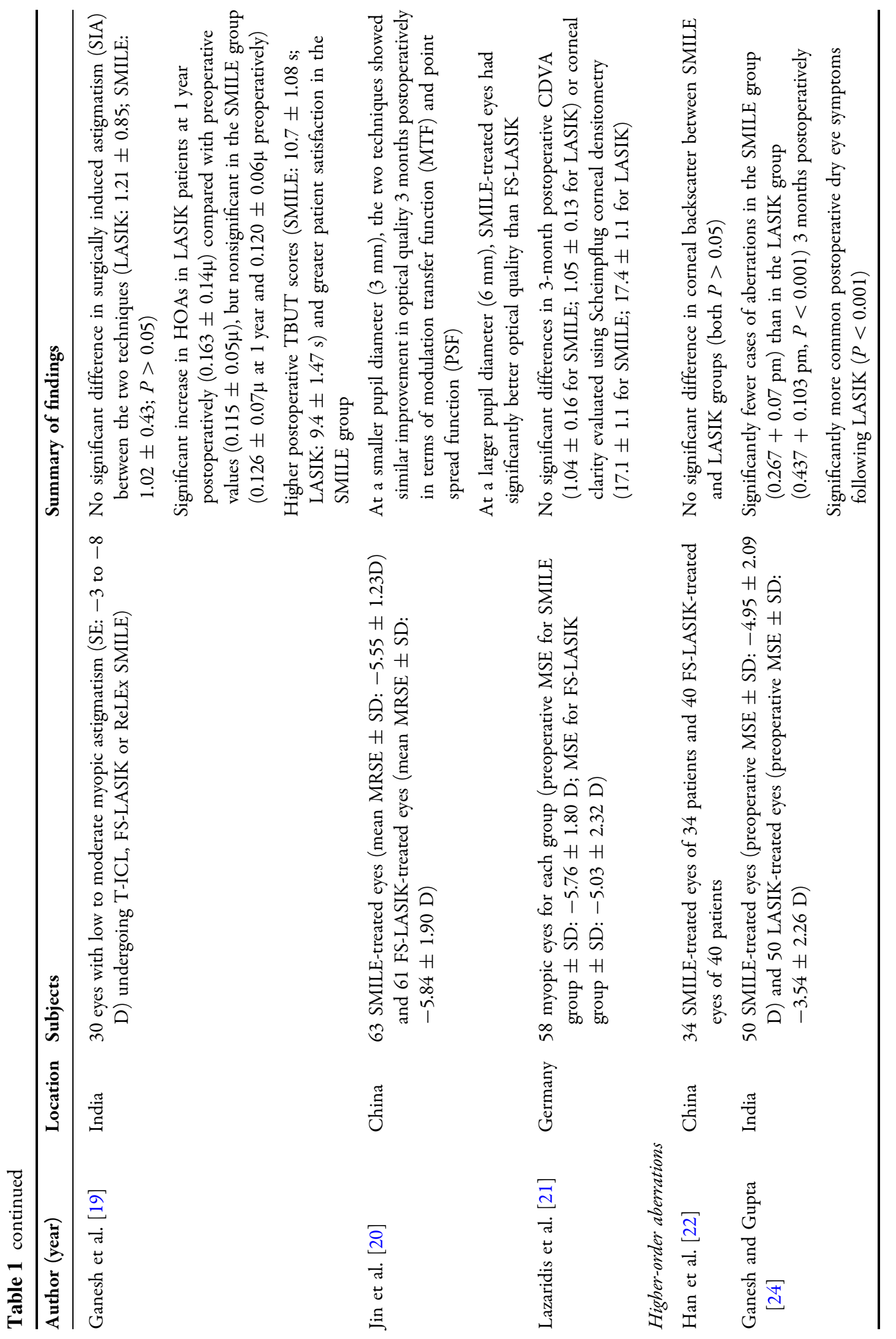




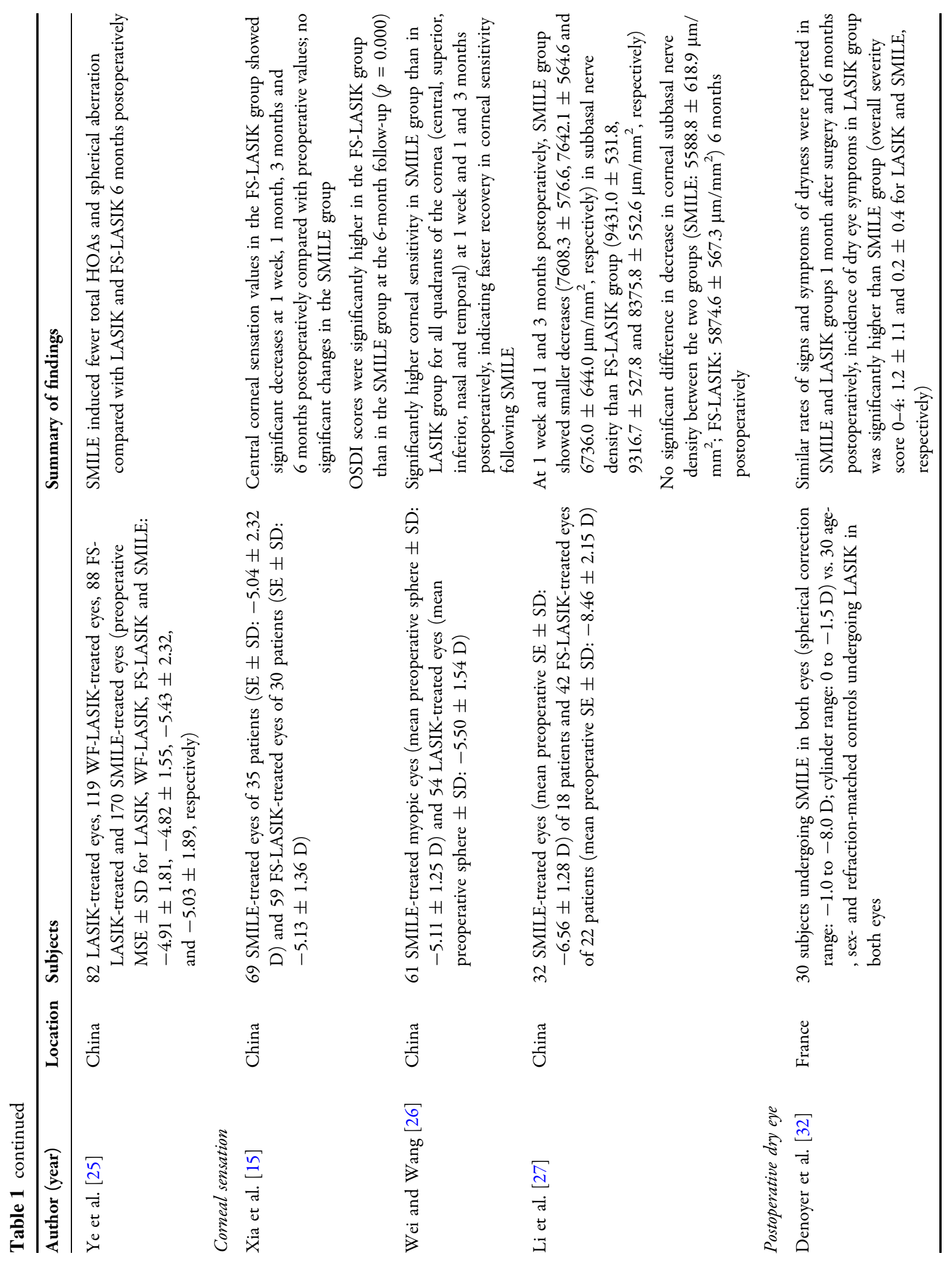




\section{RESULTS}

The majority of published observational studies have demonstrated that SMILE and LASIK, including those with custom ablation profiles for the latter, are comparable in terms of clinical efficacy, safety profiles and refractive accuracy in the treatment of both myopia and myopic astigmatism. The results from the studies were classified according to visual outcomes, HOAs, corneal sensitivity and post-treatment ocular surface disease/dry eye disease for analysis (see Table 1).

\section{Visual Outcomes}

In a 2018 Japanese retrospective case-control study on patients with moderate myopia and myopic astigmatism, Kataoka et al. compared 3-month postoperative visual outcomes between 34 eyes of 23 patients treated with SMILE (mean preoperative manifest refractive sphere: $-4.52 \pm 0.54 \mathrm{D}$; mean preoperative manifest refractive cylinder: $-0.33 \pm 0.38 \mathrm{D})$ and 34 eyes of 24 patients treated with LASIK $(-4.45 \pm 0.61 \mathrm{D} ;-0.45 \pm 0.44 \mathrm{D})$. The authors found no significant differences in postoperative uncorrected distance visual acuity (UDVA) (82.4\% and $85.3 \%$ of patients with $20 / 13$ or better UDVA for SMILE and LASIK, respectively) or corrected distance visual acuity (CDVA) (97.06\% and $100 \%$ of patients with $20 / 13$ or better CDVA for SMILE and LASIK, respectively) between groups, showing that both procedures were highly effective [7]. A retrospective study including 68 SMILE-treated eyes of 35 patients in the United States with myopia or myopic astigmatism (preoperative manifest refractive sphere and cylinder ranged from -7.50 to -2.75 $\mathrm{D}$ and -0.75 to $0.00 \mathrm{D}$, respectively) showed that, although visual outcomes for SMILE (74\% of eyes with 20/20 UDVA or better) were superior to first-generation LASIK [SVS Apex Plus, VISX STAR S2, Nidek EC-5000 (2000)] from 1999 to $2000(51 \%)$, they were inferior to the latest generation (Nidek EC-5000 (2013), Alcon Contoura, VISX iDesign) from 2013 to 2016 (89\%) [8]. The advanced generation of LASIK platforms, which can be used in wavefront- 
guided or topography-guided treatments, have advantages over the early ones, including higher pulse frequency, improved eye tracking system and greater accuracy in measuring refractive error and aberrations of the eye. However, another retrospective study in China comparing SMILE with wavefront-guided femtosecond LASIK (WFG FS-LASIK) in 65 myopic eyes (mean preoperative sphere \pm SD: $-4.20 \pm 2.65 \mathrm{D})$ found no significant differences in visual outcomes in terms of UDVA and CDVA $\quad$ (mean \pm SD: $\quad-0.02 \pm 0.07$ and $-0.04 \pm 0.22$, respectively, for WFG FS-LASIK; $-0.01 \pm 0.06$ and $-0.04 \pm 0.04$, respectively, for SMILE) [9]. A total of 60 patients with myopia or myopic astigmatism [manifest refraction spherical equivalent (MRSE) ranged from -1.25 to $-8.5 \mathrm{D}$ ] were enrolled in a study conducted by El-Mayah et al. in Egypt. Again, no statistically significant differences in CDVA were found between the two procedures at 3 months postoperatively, and the mean safety and efficacy indices for those in the SMILE group $(1.1060 .14 ; 1.0260 .17)$ were similar to those in the LASIK group $(1.1260 .15 ; 1.0760 .20)$ as well [10]. The study found that the refractive error was within $\pm 0.5 \mathrm{D}$ of target refraction in $93 \%$ of treated eyes in both groups 3 months after surgery [10], in agreement with the findings of other studies comparing the two procedures, which demonstrated high refractive accuracy and clinical efficacy for both treatments [11-13]. Furthermore, the two techniques were found to be equally safe, as no patient had a loss of more than two lines of best-corrected visual acuity (BCVA) throughout the 6-month follow-up period or suffered from significant postoperative complications such as epithelial defect, lamellar keratitis or epithelial implantation. However, in terms of speed of postoperative visual acuity improvement, the SMILE group reached values of $1.3 \pm 0.2$ at 3 months postoperatively, while the LASIK group reached this landmark already 1 week after surgery. This finding has been confirmed by other studies that also demonstrated a slower recovery rate in terms of postoperative visual acuity in SMILE-treated eyes compared with LASIK-treated eyes $[12,14]$. Xia et al. suggested that mechanical damage to the surrounding stromal tissue during lenticule dissection and extraction in SMILE may be a significant contributing factor in the slower visual recovery process [15]. In making inferences from these results, however, it is important to note that most published studies comparing the two procedures focused on patients with mild to moderate myopia. There are limited data on the comparative efficacy between procedures with respect to myopia severity. A few studies have investigated SMILE efficacy in patients with high myopia (6.0D myopia or more), which have shown good visual outcomes and safety profiles in treated eyes [16-18]. However, one prospective study including 165 eyes of 86 patients in China reported a trend towards undercorrection by SMILE in eyes with myopia greater than $6.0 \mathrm{D}$ [17].

In terms of surgically induced astigmatism (SIA), one study in India including 30 eyes with low to moderate myopic astigmatism reported that there was no statistically significant difference between the two techniques (LASIK: $1.21 \pm 0.85$; SMILE: $1.02 \pm 0.43 ; P>0.05)$ [19]. However, a study in Finland found that the SMILE group (100 eyes with preoperative SE ranging from -1.38 to $-8.25 \mathrm{D}$ ) had more postoperative astigmatism than the FS-LASIKtreated group (200 eyes with preoperative SE ranging from -0.63 to $-11.63 \mathrm{D})$ [11]. Thus the results are inconclusive with regard to which technique is more likely to induce astigmatism, and additional evidence from prospective studies and randomized controlled trials is needed.

Although SMILE and LASIK have shown comparable visual outcomes, there are a number of potential advantages with the SMILE technique. One study suggested that SMILE has greater refractive power correction efficiency than LASIK in the peripheral cornea [7]. Maintenance ratios at the eighth to tenth analysis points on the cornea in the SMILE group (8th, $83.71 \% \pm 8.44 \% ; \quad 9$ th, $74.03 \% \pm 7.9 \%$; and 10th, $63.25 \% \pm 8.06 \%)$ were significantly higher than those in the LASIK group (8th, $79.86 \% \pm 5.83 \% ; 9$ th, $70.44 \% \pm 6.11 \%$; and 10 th, $57.7 \% \pm 6.37 \%$ ). This may be explained by differences in surgical techniques. Additionally, a recent prospective clinical study, which included 123 eyes of 63 patients in China (mean 
preoperative MRSE \pm SD: $-5.55 \pm 1.23$ D), demonstrated that in the early postoperative phase up to 3 months, even though both techniques showed great improvement in optical quality at a small pupil diameter, SMILE showed better optical quality than FS-LASIK in eyes with larger pupil diameters, which may lead to better night vision with the SMILE procedure than with LASIK. [20].

The effects of surgery on corneal transparency were also examined in some studies. Corneal transparency can be impaired by corneal refractive surgery, which may hinder the passage of light rays and thus cause backward scattering of light. The two procedures demonstrated similar effects on corneal transparency. In a study comparing 58 SMILE-treated myopic eyes with 58 LASIK-treated eyes in China, no significant differences were found in 3-month postoperative CDVA (1.04 \pm 0.16 for SMILE; $1.05 \pm 0.13$ for LASIK) or corneal clarity evaluated using Scheimpflug corneal densitometry $(17.1 \pm 1.1$ for SMILE; $17.4 \pm 1.1$ for LASIK) [21]. A 3-year postoperative follow-up of two groups of myopic patients in China who underwent SMILE and LASIK (34 and 40 eyes, respectively) detected no significant difference in corneal backscatter as well [22].

Overall, LASIK had an advantage in terms of significantly faster postoperative visual recovery, but the two techniques had comparable visual outcomes from 3 months after surgery onward. Furthermore, there was evidence that eyes with large pupil diameters had better visual outcomes after SMILE than after LASIK treatment.

\section{Higher-Order Aberrations}

HOAs include vertical and horizontal coma, spherical aberration and trefoils, and are linked to problems with night vision, presenting as glare, haze and halos [13, 23]. Comparing SMILE and LASIK, studies have shown equivalence-if not superiority of the former-in their minimization of post-surgical HOAs.

A number of studies reported no significant difference in the levels of induced total HOAs between SMILE-treated and LASIK-treated eyes.
A study conducted by El-Mayah et al. reported no statistically significant differences in the change in higher-order root mean square values (RMS) (P1/40.2000), primary coma RMS (P1/ 40.0589) or spherical aberrations (P1/40.0543), but changes in the former two parameters were greater in the SMILE group, while the FS-LASIK group showed greater changes in the latter [10]. Chen et al. compared the occurrence of ocular HOAs in eyes treated with SMILE and WFG FSLASIK, which is the gold standard for treating HOAs [9]. While there was an increase in total HOAs 3 months after surgery compared with preoperative values for both groups, no significant differences postoperatively in overall ocular aberrations trefoil, horizontal coma, spherical aberration or total HOAs were found. Only vertical coma values were observed to be higher in the SMILE group $(0.163 \pm 0.093)$ than in the WFG FS-LASIK group $(0.116 \pm 0.077)$, which may be explained by the fact that no iris registration or eye tracker was used for SMILE in this study, thereby reducing accuracy in centration, and that corneal wound-healing responses for SMILE and WFG FS-LASIK are different.

However, other studies have reported a lower occurrence of HOAs in SMILE-treated patients compared with LASIK-treated patients. A recent study reported a significant increase in HOAs in LASIK patients at 1 year $(0.163 \pm 0.14 \mu)$ compared with preoperative values $(0.115 \pm 0.05 \mu)$, while the increase in these values was not statistically significant in the SMILE group $(0.126 \pm 0.07 \mu$ at 1 year and $0.120 \pm 0.06 \mu$ preoperatively) [19]. In a study in a speciality eye hospital in India, Ganesh and Gupta also reported a significantly smaller number of cases of aberrations in the SMILE group (50 eyes; mean preoperative $\mathrm{SE} \pm \mathrm{SD}:-4.95 \pm 2.09 \mathrm{D})$ than in the LASIK group (50 eyes; mean preoperative $\mathrm{SE} \pm \mathrm{SD}:-3.54 \pm 2.26$ D) [24]. In another study in China including 459 eyes of 230 patients with myopic or myopic astigmatism errors less than $-10.0 \mathrm{D}$ spherical SE, SMILE induced fewer total HOAs and spherical aberrations compared with LASIK and FS-LASIK 6 months postoperatively, possibly because a lower corneal wound-healing response is induced by the small incision in SMILE, despite 
more horizontal coma and vertical coma induced when compared with WF-LASIK [25].

\section{Corneal Sensitivity}

Most studies have demonstrated that corneal sensitivity is reduced after refractive surgery. SMILE-treated eyes tend to recover to preoperative levels sooner than LASIK-treated eyes.

In one study, central corneal sensation values in the FS-LASIK group were significantly decreased at 1 week, 1 month, 3 months and 6 months postoperatively compared with preoperative values [15]. However, central corneal sensation was not significantly changed in the SMILE group at any time point postoperatively compared with preoperative measurements, and quickly returned to preoperative values at 1 month post-surgery. In a study conducted by Wei and Wang in China, significantly higher corneal sensitivity was seen in the SMILE group (61 eyes; mean preoperative sphere \pm SD: $-5.11 \pm 1.25 \mathrm{D}$ ) than the LASIK group (54 eyes; mean preoperative sphere $\pm \mathrm{SD}:-5.50 \pm 1.54$ D) in all quadrants of the cornea (central, superior, inferior, nasal and temporal) at 1 week and 1 and 3 months postoperatively, indicating faster recovery in corneal sensitivity for SMILEtreated myopic eyes, which had comparable preoperative and postoperative corneal sensitivity [26]. These results were supported by another study looking at corneal reinnervation after refractive surgery in 74 eyes of 40 patients in China. At 1 week and 1 and 3 months postoperatively, the SMILE group (mean preoperative SE \pm SD: $-6.56 \pm 1.28$ D) showed smaller decreases (7608.3 576.6, $7642.1 \quad 564.6$ and $6736.0644 .0 \mu \mathrm{m} / \mathrm{mm}^{2}$, respectively) in subbasal nerve density than the FS-LASIK group (mean preoperative $\mathrm{SE} \pm \mathrm{SD}: \quad-8.46 \pm 2.15 \quad \mathrm{D}$; $9431.0 \pm 531.8, \quad 9316.7 \pm 527.8 \quad$ and $8375.8 \pm 552.6 \mu \mathrm{m} / \mathrm{mm}^{2}$, respectively). However, no significant difference in the decrease in corneal subbasal nerve density was observed between the two groups (SMILE: $5588.8 \pm 618.9 \mu \mathrm{m} / \mathrm{mm}^{2}$; $\quad$ FS-LASIK: $5874.6 \pm 567.3 \mu \mathrm{m} / \mathrm{mm}^{2}$ ) 6 months postoperatively [27].
Previous studies have shown that recovery of corneal sensation to preoperative levels takes about 3-6 months after LASIK [28]. It is believed that since SMILE does not involve the creation of a corneal flap, better preservation of corneal subbasal and stromal nerves can be achieved when compared with LASIK, resulting in better postoperative corneal sensitivity [3, 29].

\section{Ocular Surface Disease}

Dry eye and corneal neuropathic pain are one of the most commonly reported complaints after laser refractive surgery. In severe cases, patients may even require referral to tertiary eye centers for effective symptom relief [30]. Dry eye not only lowers patients' quality of life, but also damages the ocular surface and gives rise to various symptoms of ocular discomfort associated with visual disturbance, thus impeding good visual recovery [31-33]. In general, studies agree that SMILE results in less severe postoperative dry eye symptoms than LASIK.

In a study conducted in France including 60 European subjects (spherical correction range: -1.0 to $-8.0 \mathrm{D}$; cylinder range: 0 to -1.5 D), similar rate of signs and symptoms of dryness were reported between SMILE and LASIK groups 1 month after surgery, but the rate was significantly higher for LASIK than SMILE 6 months postoperatively [32]. A retrospective analysis of 68 SMILE-treated eyes showed that dry eyes were most frequently reported early in the postoperative period and decreased in severity with time, with dry eyes reported in $100 \%$ and $88 \%$ at the 1 -week and 1-month intervals, respectively, returning to baseline level of $45 \%$ at 3 months [8]. Ganesh et al. included 30 eyes of 30 patients with low to moderate myopic astigmatism, and found a significant decrease in tear breakup time (TBUT) scores $(9.4 \pm 1.47 \mathrm{~s})$ in the FS-LASIK group at 1 year $(P=0.00)$, leading to lower patient satisfaction due to dry eye symptoms, compared with the higher TBUT scores $(10.7 \pm 1.08 \mathrm{~s})$, which were comparable to preoperative values $(11.26 \pm 1.22 \mathrm{~s})$, and excellent satisfaction among SMILE-treated patients [19]. Other relevant studies showed similar results [10, 15, 24]. 
A study investigating early ocular surface changes after the two procedures revealed that in terms of corneal fluorescein staining, SMILE patients recovered to preoperative levels by 1 week after surgery, as opposed to 1 month postoperatively in LASIK patients. Additionally, a quicker recovery in TBUT can be seen in SMILE (1 month) than in FS-LASIK patients (3 month). Assessment of dry eye symptoms using the Ocular Surface Disease Index (OSDI) questionnaire also revealed that OSDI scores in the SMILE and LASIK groups reached preoperative levels by 1 month and 3 months after surgery, respectively, further suggesting that quicker ocular surface recovery can be achieved with SMILE [34]. Tear film mediators are believed to play a role in corneal wound healing, and levels of IL-6, which is associated with damage to the ocular surface after refractive surgery, were lower in SMILE-treated than in LASIK-treated eyes.

Corneal nerves carry afferent signals important for maintaining the ocular surface lacrimal gland feedback system for tear production [35]. It has been hypothesized that transection of corneal nerves during refractive surgery is a major contributor to postoperative dry eye symptoms [36]. In SMILE, since the creation of a corneal flap is not involved, damage to the anterior stromal nerve plexus is significantly less than that in FS-LASIK, in which flap creation results in a greater number of corneal nerves around the ring being severed; hence fewer dry eye symptoms are seen postoperatively with SMILE [32, 37]. Based on the aforementioned studies, it is reasonable to conclude that SMILE induces fewer dry eye symptoms than LASIK in a comparable population.

\section{DISCUSSION}

A large number of studies have compared the clinical outcomes of LASIK and SMILE. However, there are currently very few randomized controlled trials comparing the two techniques. Considering that most evidence is from observational studies, selection bias may result as patients are selected for a particular laser refractive technique based on preferences and ocular factors. There is also a lack of data from registries or multi-surgeon data sets. Many of the outcomes are reported from single-surgeon data sets or from experts, which may have skewed the results towards better outcomes. Moreover, heterogeneity is observed in the results of these studies. This may be explained by the fact that, in contrast to SMILE, there is no single standard LASIK technique. There are a number of different femtosecond lasers that cut different flap thicknesses, as well as different excimer lasers with varying accuracy. It is also important to note that SMILE was compared with different types of LASIK across different studies. Conventional LASIK and "custom" LASIK (including WFG and topography-guided LASIK) differ in various aspects including technology, effectiveness and accuracy, which may account for the heterogenous results. Nonetheless, based on the existing literature, it is reasonable to conclude that both SMILE and LASIK achieve very good outcomes for the correction of mild to moderate myopia.

There are also some limitations of the studies presented in this review, one being that most studies had a relatively short follow-up period, ranging from 1 to 6 months post-surgery. Less information was acquired based on a longer follow-up period for the assessment of longterm clinical outcomes of SMILE and LASIK. Another major flaw is that a number of femtosecond and excimer laser platforms are available for LASIK, and various platforms were used in these studies, while VisuMax is the only SMILE platform at present. This likely explains the discrepancy in the results. There are also a limited number of studies comparing the outcomes of SMILE and LASIK based on the severity of myopia. Additionally, some studies had a small sample size, and thus reduced statistical power. Future prospective randomized controlled trials with a larger sample size and longer follow-up period may help to better determine the clinical outcomes of SMILE and LASIK, which will facilitate decision-making by doctors and patients regarding the choice of refractive surgery. While our review article aimed to summarize and compare results from published studies, we did not perform a meta-analysis of the 22 identified original articles. This will 
certainly be a worthwhile endeavor as the number of published studies on this subject increases. Finally, only Entrez PubMed was used for our literature search, and other search engines including Google Scholar, Cochrane database, EMBASE and SCOPUS were not employed.

A major limitation of the VisuMax platform is the lack of an automated cyclotorsion-compensation system in the SMILE platform at present. Cyclotorsional error of only a few degrees can contribute to undercorrection of astigmatism [6]. Additional cyclotorsion has been reported as a result of the docking and suction maneuver during SMILE treatment, thus potentially affecting axis alignment in astigmatism treatment [38]. For patients with high astigmatism, treatment with an excimerlaser platform with cyclotorsion compensation is thus recommended.

\section{CONCLUSION}

In conclusion, SMILE and LASIK have comparable safety, efficacy and predictability in treating myopia and myopic astigmatism. Despite similar effects on corneal clarity, along with some potential advantages of SMILE such as greater refractive power correction efficiency in the peripheral cornea, recovery of visual acuity may be slower in SMILE-treated than in LASIK-treated eyes. In addition, the incidence of HOAs with SMILE tends to be similar-or even lower, as some studies suggested-than with LASIK treatment. Regarding corneal sensitivity, SMILEtreated eyes recover more quickly to preoperative levels than LASIK-treated eyes. Both procedures may cause postoperative dry eye symptoms, but the severity is lower with SMILE. However, SMILE has its limitations as well, including the inability to treat hyperopia and the lack of a cyclotorsioncompensation system at present.

\section{ACKNOWLEDGEMENTS}

Funding. No funding or sponsorship was received for this study or publication of this article. The Rapid Service Fee was funded by the authors. All authors had full access to the articles reviewed in this manuscript and take complete responsibility for the integrity and accuracy of this manuscript.

Authorship. All named authors meet the International Committee of Medical Journal Editors (ICMJE) criteria for authorship for this article, take responsibility for the integrity of the work as a whole, and have given their approval for this version to be published.

Disclosures. Yumi Tsz-Ying Lau, Kendrick Co Shih, Ryan Hin-Kai Tse, Tommy Chung-Yan Chan and Vishal Jhanji have nothing to declare.

Compliance with Ethics Guidelines. This article is based on previously conducted studies and does not contain any studies with human participants or animals performed by any of the authors.

Data Availability. The authors agree to make all materials, data and associated protocols promptly available to readers without undue qualifications in material transfer agreements.

Open Access. This article is distributed under the terms of the Creative Commons Attribution-NonCommercial 4.0 International License (http://creativecommons.org/licenses/ by-nc/4.0/), which permits any noncommercial use, distribution, and reproduction in any medium, provided you give appropriate credit to the original author(s) and the source, provide a link to the Creative Commons license, and indicate if changes were made.

\section{REFERENCES}

1. Chen S, Feng Y, Stojanovic A, Jankov MR, Wang Q. IntraLase femtosecond laser vs mechanical microkeratomes in LASIK for myopia: a systematic review and meta-analysis. J Refract Surg (Thorofare, NJ : 1995). 2012;28(1):15-24. 
2. Dupps WJ Jr. LASIK outcomes: how are we doing and can we do better? J Cataract Refract Surg. 2016;42(8):1109-10.

3. Sekundo W, Kunert KS, Blum M. Small incision corneal refractive surgery using the small incision lenticule extraction (SMILE) procedure for the correction of myopia and myopic astigmatism: results of a 6 month prospective study. Br J Ophthalmol. 2011;95(3):335-9.

4. Bharti S, Bains HS. Active cyclotorsion error correction during LASIK for myopia and myopic astigmatism with the NIDEK EC-5000 CX III laser. J Refract Surg (Thorofare, NJ : 1995). 2007;23(9 Suppl):S1041-5.

5. Febbraro JL, Koch DD, Khan HN, Saad A, Gatinel D. Detection of static cyclotorsion and compensation for dynamic cyclotorsion in laser in situ keratomileusis. J Cataract Refract Surg. 2010;36(10):1718-23.

6. Alio Del Barrio JL, Vargas V, Al-Shymali O, Alio JL. Small incision lenticule extraction (SMILE) in the correction of myopic astigmatism: outcomes and limitations-an update. Eye Vision (London, England). 2017;4:26.

7. Kataoka T, Nishida T, Murata A, et al. Controlmatched comparison of refractive and visual outcomes between small incision lenticule extraction and femtosecond laser-assisted LASIK. Clin Ophthalmol (Auckland, NZ). 2018;12:865-73.

8. Moshirfar M, Murri MS, Shah TJ, et al. Initial singlesite surgical experience with SMILE: a comparison of results to FDA SMILE, and the earliest and latest generation of LASIK. Ophthalmol Ther. 2018;7:347-60.

9. Chen X, Wang Y, Zhang J, Yang SN, Li X, Zhang L. Comparison of ocular higher-order aberrations after SMILE and wavefront-guided femtosecond LASIK for myopia. BMC Ophthalmol. 2017;17(1):42.

10. El-Mayah E, Anis M, Salem M, Pinero D, Hosny M. Comparison between Q-adjusted lasik and smallincision lenticule extraction for correction of myopia and myopic astigmatism. Eye \& contact lens. 2018;44:S426-32.

11. Pietila J, Huhtala A, Makinen P, et al. Uncorrected visual acuity, postoperative astigmatism, and dry eye symptoms are major determinants of patient satisfaction: a comparative, real-life study of femtosecond laser in situ keratomileusis and small incision lenticule extraction for myopia. Clin Ophthalmol (Auckland, NZ). 2018;12:1741-55.

12. Liu M, Chen Y, Wang D, et al. Clinical outcomes after SMILE and femtosecond laser-assisted LASIK for myopia and myopic astigmatism: a prospective randomized comparative study. Cornea. 2016;35(2):210-6.

13. Lin $\mathrm{F}, \mathrm{Xu} \mathrm{Y}$, Yang $\mathrm{Y}$. Comparison of the visual results after SMILE and femtosecond laser-assisted LASIK for myopia. J Refract Surg (Thorofare, NJ : 1995). 2014;30(4):248-54.

14. Chiche A, Trinh L, Saada O, et al. Early recovery of quality of vision and optical performance after refractive surgery: small-incision lenticule extraction versus laser in situ keratomileusis. J Cataract Refract Surg. 2018;44(9):1073-9.

15. Xia L, Zhang J, Wu J, Yu K. Comparison of corneal biological healing after femtosecond LASIK and small incision lenticule extraction procedure. Curr Eye Res. 2016;41(9):1202-8.

16. Xia LK, Ma J, Liu HN, Shi C, Huang Q. Three-year results of small incision lenticule extraction and wavefront-guided femtosecond laser-assisted laser in situ keratomileusis for correction of high myopia and myopic astigmatism. Int $\mathrm{J}$ Ophthalmol. 2018;11(3):470-7.

17. Jin HY, Wan T, Wu F, Yao K. Comparison of visual results and higher-order aberrations after small incision lenticule extraction (SMILE): high myopia vs. mild to moderate myopia. BMC Ophthalmol. 2017;17(1):118.

18. Kim JR, Kim BK, Mun SJ, Chung YT, Kim HS. Oneyear outcomes of small-incision lenticule extraction (SMILE): mild to moderate myopia vs. high myopia. BMC Ophthalmol. 2015;15:59.

19. Ganesh S, Brar S, Pawar A. Matched population comparison of visual outcomes and patient satisfaction between 3 modalities for the correction of low to moderate myopic astigmatism. Clin Ophthalmol (Auckland, NZ). 2017;11:1253-63.

20. Jin $\mathrm{Y}$, Wang $\mathrm{Y}, \mathrm{Xu} \mathrm{L}$, et al. Comparison of the optical quality between small incision lenticule extraction and femtosecond laser LASIK. J Ophthalmol. 2016;2016:2507973.

21. Lazaridis A, Droutsas K, Sekundo W, Petrak M, Schulze S. Corneal clarity and visual outcomes after small-incision lenticule extraction and comparison to femtosecond laser-assisted in situ keratomileusis. J Ophthalmol. 2017;2017:5646390.

22. Han T, Zhao J, Shen Y, Chen Y, Tian M, Zhou X. A three-year observation of corneal backscatter after small incision lenticule extraction (SMILE). J Refract Sur (Thorofare, NJ: 1995). 2017;33(6):377-82.

23. Agarwal S, Thornell E, Hodge C, Sutton G, Hughes P. Visual outcomes and higher order aberrations 
following LASIK on eyes with low myopia and astigmatism. Open Ophthalmol J. 2018;12:84-93.

24. Ganesh S, Gupta R. Comparison of visual and refractive outcomes following femtosecond laserassisted lasik with smile in patients with myopia or myopic astigmatism. J Refract Surg (Thorofare, NJ: 1995). 2014;30(9):590-6.

25. Ye MJ, Liu CY, Liao RF, Gu ZY, Zhao BY, Liao Y. SMILE and wavefront-guided LASIK out-compete other refractive surgeries in ameliorating the induction of high-order aberrations in anterior corneal surface. J Ophthalmol. 2016;2016:8702162.

26. Wei S, Wang Y. Comparison of corneal sensitivity between FS-LASIK and femtosecond lenticule extraction (ReLEx flex) or small-incision lenticule extraction (ReLEx smile) for myopic eyes. Graefe's Arch Clin Exp Ophthalmol. 2013;251(6):1645-54.

27. Li M, Niu L, Qin B, et al. Confocal comparison of corneal reinnervation after small incision lenticule extraction (SMILE) and femtosecond laser in situ keratomileusis (FS-LASIK). PLoS One. 2013;8(12):e81435.

28. He M, Huang W, Zhong X. Central corneal sensitivity after small incision lenticule extraction versus femtosecond laser-assisted LASIK for myopia: a meta-analysis of comparative studies. BMC Ophthalmol. 2015;15:141.

29. Ganesh S, Brar S, Arra RR. Refractive lenticule extraction small incision lenticule extraction: a new refractive surgery paradigm. Indian J Ophthalmol. 2018;66(1):10-9.

30. Wallerstein A, Jackson WB, Chambers J, Moezzi AM, Lin H, Simmons PA. Management of postLASIK dry eye: a multicenter randomized comparison of a new multi-ingredient artificial tear to carboxymethylcellulose. Clin Ophthalmol (Auckland, NZ). 2018;12:839-48.
31. Deschamps N, Ricaud X, Rabut G, Labbe A, Baudouin $C$, Denoyer A. The impact of dry eye disease on visual performance while driving. Am J Ophthalmol. 2013;156(1):184-189.e183.

32. Denoyer A, Landman E, Trinh L, Faure JF, Auclin F, Baudouin C. Dry eye disease after refractive surgery: comparative outcomes of small incision lenticule extraction versus LASIK. Ophthalmology. $2015 ; 122(4): 669-76$.

33. The definition and classification of dry eye disease: report of the Definition and Classification Subcommittee of the International Dry Eye WorkShop (2007). The Ocular Surface. 2007;5(2):75-92.

34. Gao S, Li S, Liu L, et al. Early changes in ocular surface and tear inflammatory mediators after small-incision lenticule extraction and femtosecond laser-assisted laser in situ keratomileusis. PLoS One. 2014;9(9):e107370.

35. Mathers WD. Why the eye becomes dry: a cornea and lacrimal gland feedback model. CLAO J. 2000;26(3):159-65.

36. Wilson SE, Ambrosio R. Laser in situ keratomileusisinduced neurotrophic epitheliopathy. Am J Ophthalmol. 2001;132(3):405-6.

37. Mohamed-Noriega K, Riau AK, Lwin NC, Chaurasia SS, Tan DT, Mehta JS. Early corneal nerve damage and recovery following small incision lenticule extraction (SMILE) and laser in situ keratomileusis (LASIK). Invest Ophthalmol Vis Sci. 2014;55(3):1823-34.

38. Agca A, Demirok A, Yildirim Y, et al. Refractive lenticule extraction (ReLEx) through a small incision (SMILE) for correction of myopia and myopic astigmatism: current perspectives. Clin Ophthalmol (Auckland, NZ). 2016;10:1905-12. 